\title{
Electron Scattering: A Microscope for Nuclear and Nucleon Structure
}

\section{S. Cardman*a}

${ }^{a}$ Thomas Jefferson National Accelerator Facility, 12000 Jefferson Ave., Newport News, VA, 23606, USA

We review progress and assess the outlook for investigations of the structure of nuclei and nucleons using electron scattering.

\section{INTRODUCTION}

The field of electron scattering studies of nuclear physics is approaching its $50^{\text {th }}$ birthday; the first experiment to show effects due to the finite size of the nucleus [1] was published 47 years ago, and 44 years have passed since the pioneering experiment of Hofstadter and McAllister [2], which first revealed the finite size of the proton. The field is being revitalized (for a second time) through major improvements in accelerator and detector technology. A third generation of facilities is providing data of unprecedented quality and detail addressing issues at the foundations of our field. It is the aim of this paper to provide a sense of the excitement and opportunities through examples of the quality of the data emerging from these new facilities and its impact on our understanding of the fundamental underpinnings of nuclear physics.

Electron scattering is the tool of choice for the precise investigation of the spatial structure of nuclei. The precision arises from the well-known characteristics of the electromagnetic interaction: 1) the interaction (QED) is well-understood, so measurements can be interpreted directly in terms of the structure of the system under study; 2) the interaction is weak, so measurements can be made without greatly disturbing the system's structure; and 3) by varying the momentum transferred from the electron to the target for fixed energy transfer we can directly map out its charge and current densities, and the transition densities associated with its excitation.

An example of the power of electron scattering is provided in Fig. 1, which compares the charge densities for nuclei from ${ }^{4} \mathrm{He}$ through ${ }^{208} \mathrm{~Pb}$ as inferred from elastic electron scattering experiments with the predictions of mean field theory. Note that electron scattering has determined the charge density with percent-level accuracy throughout the nuclear volume. Because the electron-nucleus interaction is well understood, the differences between experiment and theory can be interpreted unambiguously as failings of the theory.

The NIKHEF ${ }^{208} \mathrm{~Pb}\left(e, e^{\prime} p\right)$ data [4] shown in Fig. 2 illustrate the additional level of detailed information that becomes available when experiments involving the coincident detection of nuclear reaction products can be carried out with electromagnetic probes. In

*Supported by the U. S. Department of Energy under Contract No. DE-AC05-84ER40150. 


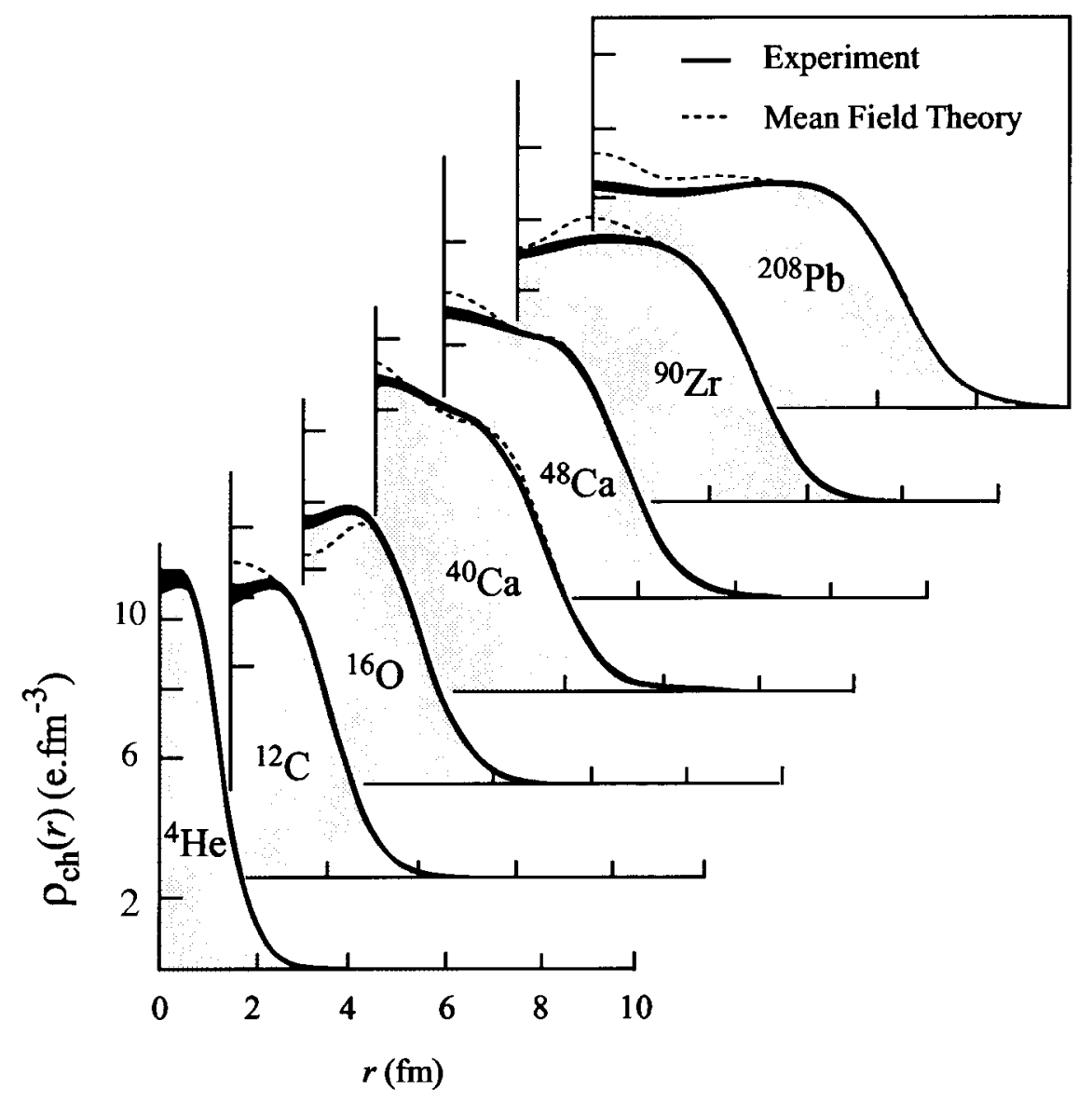

Figure 1. The ground-state charge densities for a series of doubly-magic nuclei inferred from elastic electron scattering experiments are compared with the predictions of mean field theory [3].

this experiment the inelastically scattered electron deposits a known amount of energy and momentum in the nucleus, and by measuring the distributions of the coincident knockedout protons about the momentum transfer axis one can determine the binding energies and reconstruct the wavefunctions of the protons in the nucleus shell by shell.

\section{SOME OPEN QUESTIONS IN NUCLEAR PHYSICS}

There are many important questions that must be answered to complete our understanding of the physics of the nucleus. In the traditional language of the field, some of the key questions can be stated as follows:

- What are the limitations of conventional descriptions of finite nuclei? (I.e. descriptions based on the mean field approximation, the shell model, and ab initio calculations based on phenomenological nucleon-nucleon potentials.) 


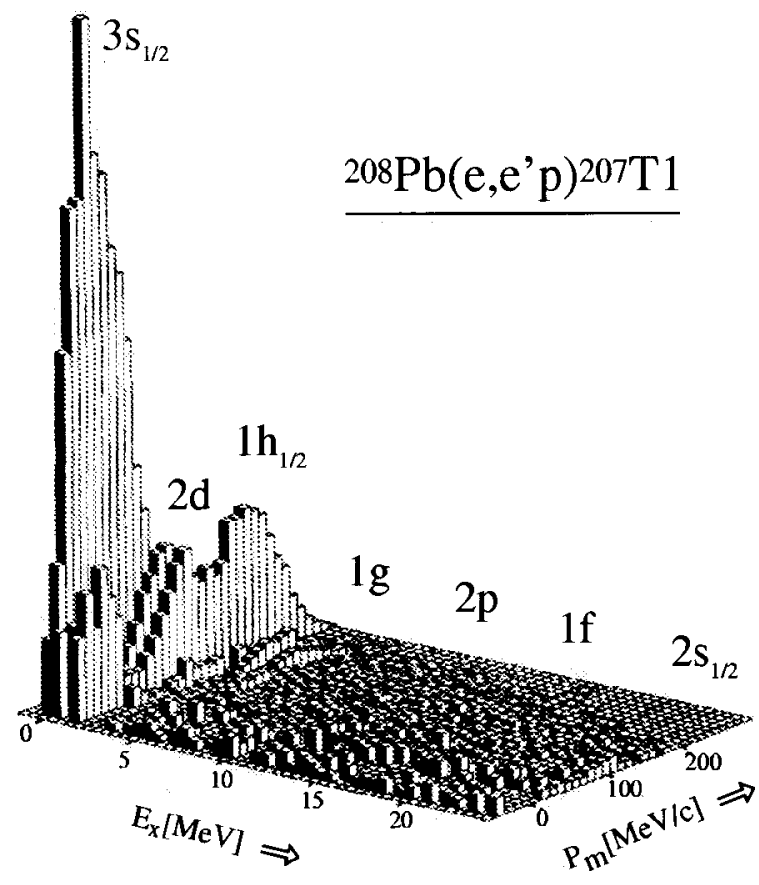

Figure 2. The coincidence cross section for ${ }^{208} \mathrm{~Pb}\left(e, e^{\prime} p\right)$ as measured at NIKHEF [4]. The data are plotted as a function of $E_{x}$, the missing energy in the residual nucleus, and $p_{M}$, the momentum of the struck proton.

- What is the short-range behavior of the strong interaction?

- What are the detailed properties of the nucleons? Do they change in the nuclear medium?

These questions can be recast from the point of view of our evolving understanding of nuclei as constructed from quarks and gluons:

- When do the underlying quark degrees of freedom become important in nuclear physics?

- How does the strong interaction arise from the residue of the QCD quark-quark interaction?

- How are the nucleons constructed from the quarks and gluons of QCD?

We know that QCD must form the basis of a deeper understanding of nuclear physics and the strong interaction - protons and neutrons are not fundamental particles, but rather are constructed from the quarks and gluons of QCD, and the "strong" interaction is really the residue of the even stronger quark-quark interaction. Understanding this connection is one of the great challenges facing nuclear physics today. The sections that 
follow provide examples of how modern electron scattering experiments are addressing these questions. Section 3 focuses on recent results in nuclear structure, while Section 4 examines progress toward our evolving understanding of nucleon structure.

\section{TOWARD THE DETAILED UNDERSTANDING OF NUCLEAR STRUCTURE}

The new generation of electron scattering coincidence experiments, underway at Darmstadt, Jefferson Lab, Mainz, MIT/Bates, and NIKHEF, is providing detailed information that will lead to a more complete understanding of conventional nuclear structure. The subsections that follow provide examples from recent experiments investigating phenomena such as:

- the short distance behavior of nucleons in nuclei and of nucleon correlations through measurements of $\left(e, e^{\prime} p\right)$ to high $q^{2}$, of two nucleon $\left[\left(e, e^{\prime} p p\right)\right.$ and $\left.\left(e, e^{\prime} p n\right)\right]$ and multinucleon knockout; and related studies via photon-induced reactions $[(\gamma, p),(\gamma, p n)$, $(\gamma, p p), \ldots]$;

- nuclear shell structure via $\left(e, e^{\prime} p\right)$ studies of deeply-bound shells and the extension of previous measurements to higher $q^{2}$ (and therefore shorter distance scales), and via high resolution hypernucleus spectroscopy;

- collective excitations such as the giant multipole resonances via reactions like $\left(e, e^{\prime} p\right)$, $\left(e, e^{\prime} n\right),\left(e, e^{\prime} \alpha\right)$, and $\left(e, e^{\prime} \gamma\right)$

\subsection{Coincidence experiments investigating correlations in nuclei}

The first generation of $\left(e, e^{\prime} p\right)$ experiments at Saclay, NIKHEF, and MIT/Bates provided substantial evidence for correlations in nuclei. One important result is shown in Fig. 3, which displays spectroscopic factors inferred from a series of these experiments; a

value of $\sim 65 \%$ is typical. The simplest explanation for the low measured spectroscopic factors is that correlations have reduced the occupancy of the low-lying valence shells [5]. Further evidence is available, e.g., from the Saclay experiment [6] on two- and three-body breakup in ${ }^{3} \mathrm{He}\left(e, e^{\prime} p\right)$.

The new generation of experiments is typified by the results of a recent experiment [8] carried out at Mainz, in which not only a knocked-out proton but also a second, correlated proton were detected in coincidence with the inelastically-scattered electron. Fig. 4 shows the excitation energy in the residual $\left({ }^{10} \mathrm{Be}\right)$ nucleus, compared with theoretical estimates for both hard core [9] and soft core [10] short range correlation (SRC) functions. The data are consistent with neither calculation. Fig. 5 shows the proton momentum spectra for cuts on the residual nucleus that define mostly $\left(1 \mathrm{p}^{2}\right)$ (left) and both $1 \mathrm{p}^{2}$ and $1 \mathrm{p} 1 \mathrm{~s}$ (right) residual states. The data lie as much as 3 orders of magnitude above the predictions of a variational monte carlo (VMC) soft core SRC calculation [10], providing direct evidence for nucleon-nucleon correlations in ${ }^{12} \mathrm{C}$.

Fig. 6 shows the measured angular correlation between the two protons, again compared with theory. The data favor calculations with a soft core SRC function, and are consistent with the effects of correlations being small; for example, a calculation with no SRC, but 


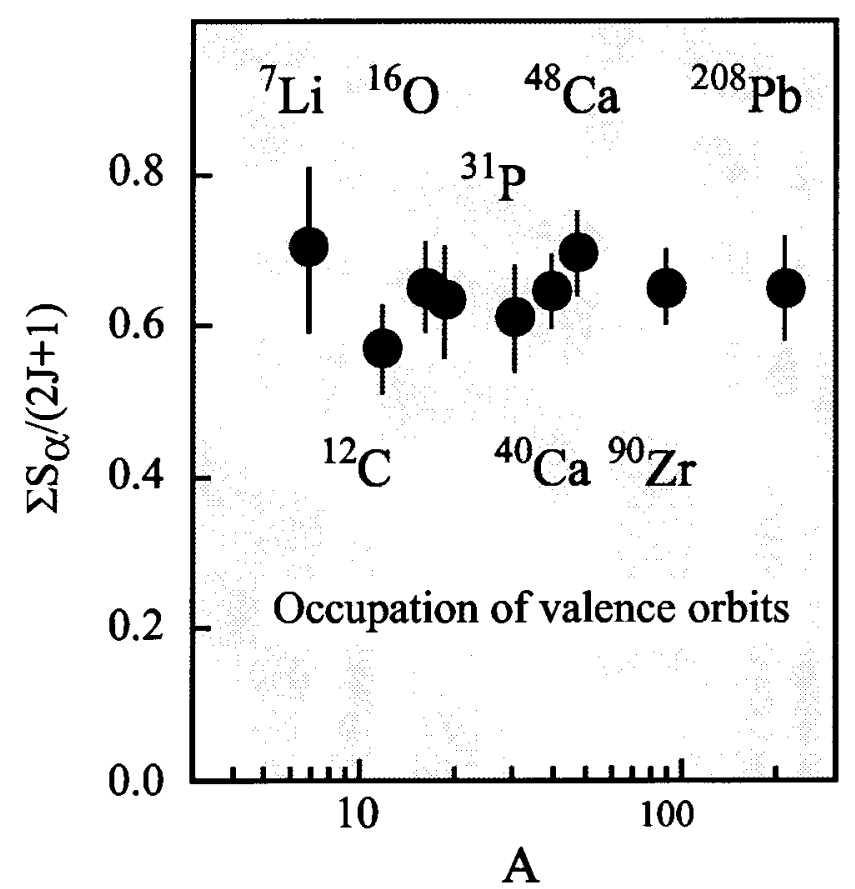

Figure 3. Spectroscopic factors inferred from a series of $\left(e, e^{\prime} p\right)$ experiments [7].

which includes he effects of delta excitation in $\left(e, e^{\prime} p\right)$, explains most of the measured cross section. Similar results have been obtained for the $p-p$ relative momentum distribution.

Fig. 7 displays similar results obtained in a recent NIKHEF measurement of ${ }^{3} \mathrm{He}\left(e, e^{\prime} p p\right)$. The theory is more reliable for ${ }^{3} \mathrm{He}$ because the properties of the 3 -body system, including its final-state interactions, can be calculated precisely. Only the $p_{M}$ distribution is shown for the NIKHEF data, but the differences between theory and experiment for other plots show results similar to those shown above for the Mainz carbon data. Note again the excess strength seen at large missing momentum in the data relative to the calculations.

A major new program examining nuclear correlations will be undertaken soon at Jefferson Lab using the CLAS detector. This instrument covers nearly half of the full $4 \pi$ solid angle and it can detect multi-nucleon emission over a broad range of momenta with full particle ID capability ( though with limited resolution compared to conventional magnetic spectrometers). Background data taken on a thin aluminum target cell during early commissioning of CLAS cleanly observed millions of $\left(e, e^{\prime} N\right),\left(e, e^{\prime} 2 N\right), \ldots$ through $\left(e, e^{\prime} 5 N\right)$ events. With this new instrument it should be possible to map out the broad features of correlated nucleon emission, and then to use this information to design selective, high resolution experiments to examine features of interest in detail.

\subsection{Few-body experiments}

Experiments on few-body systems have been a fertile ground for progress in nuclear physics because it is possible to carry out exact calculations for these systems (including final-state interactions) based on a description of the N-N interaction, predict the theoretical charge and current densities, and then compare these predictions with the precise, unambiguous measurements possible using electron scattering. Pushing both the precision 


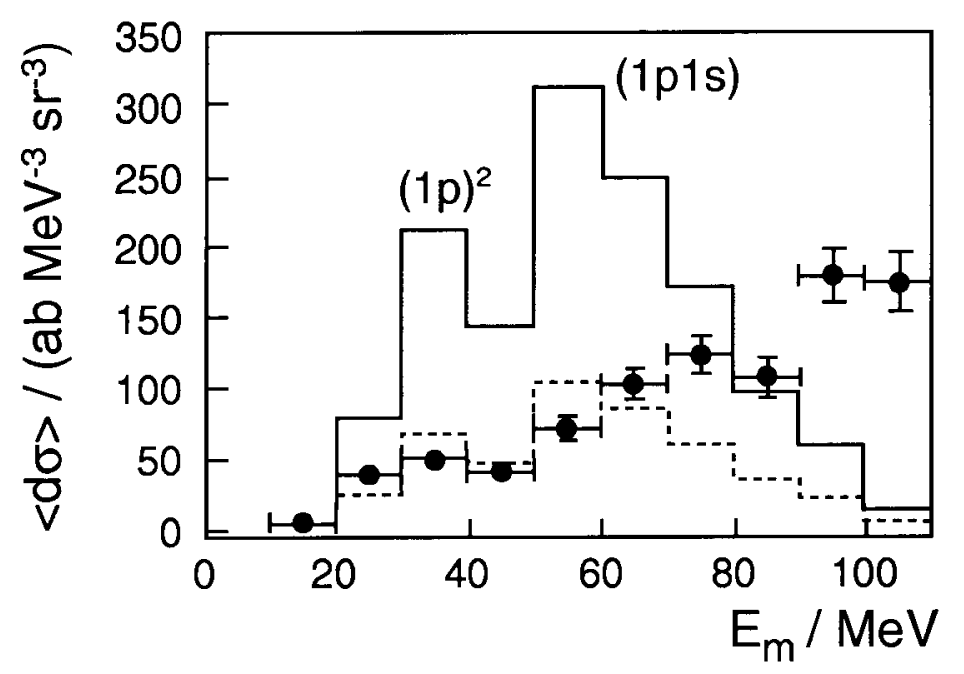

Figure $4 .{ }^{12} \mathrm{C}\left(e, e^{\prime} p p\right)$ vs $E_{M}$ as measured at Mainz [8]. The histograms display calculations using a hard core [9] (solid line) or soft core [10] (dashed line) SRC function.
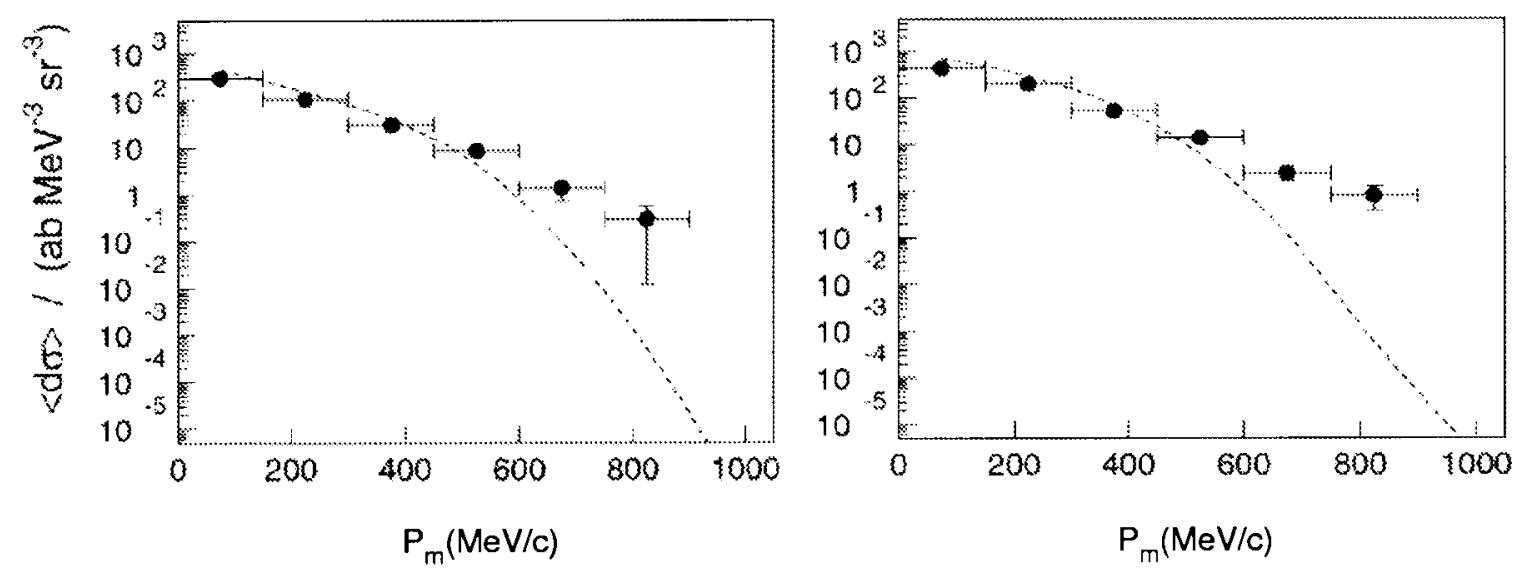

Figure $5 .{ }^{12} \mathrm{C}\left(e, e^{\prime} p p\right)$ vs $p_{M}$, for $E_{M} \leq 50 \mathrm{MeV}$ (left) and $E_{M} \leq 70 \mathrm{MeV}$ (right). Mainz data [8] are compared with calculations using a soft-core SRC function [10]. 


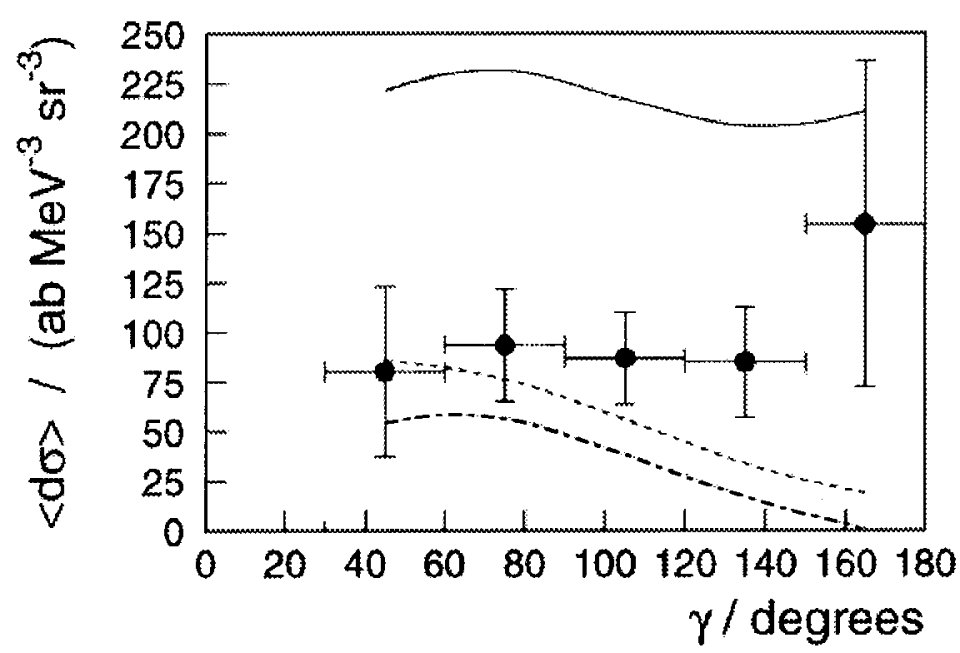

Figure 6. Proton-proton angular correlations [8] in ${ }^{12} \mathrm{C}\left(e, e^{\prime} p p\right)$ for $E_{M} \leq 70 \mathrm{MeV}$, compared with calculations using a hard-core [9] (solid curve) and a soft-core [10] (dashed curve) SRC function, and a calculation without SRC (dash-dot curve).

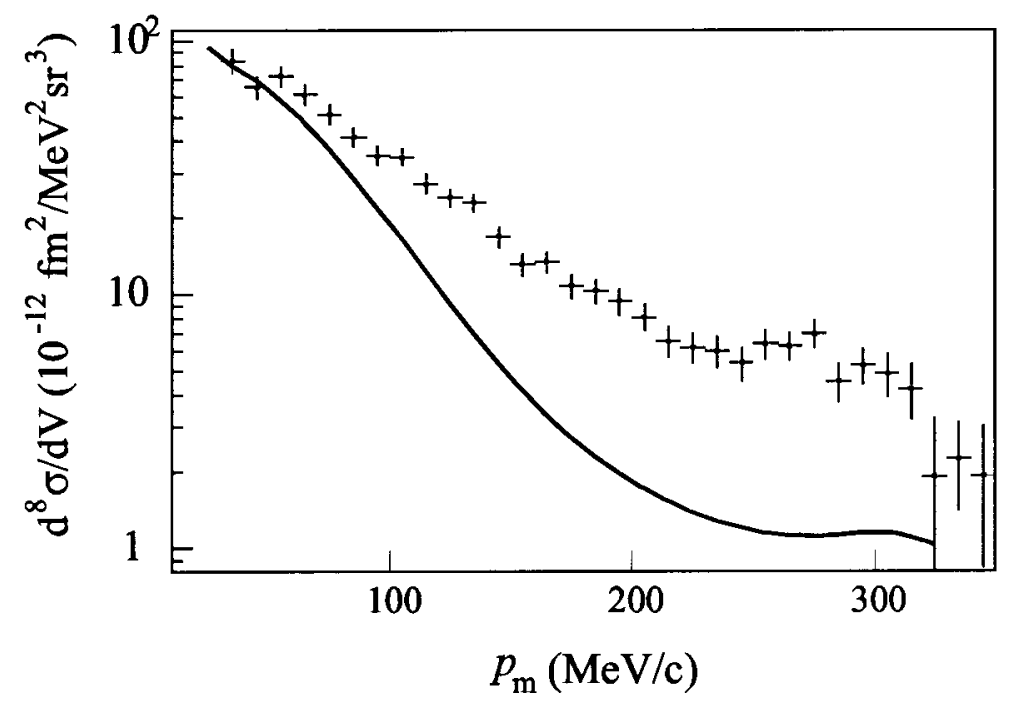

Figure 7. ${ }^{3} \mathrm{He}\left(e, e^{\prime} p p\right)$ vs $p_{M}$ : recent NIKHEF data [11] are compared with a Faddeev calculation [12] by the Bochum group using the Bonn-B potential. 
of the experiments and their spatial resolution will identify the limits of the theory.

The "classical" nuclear theory at the heart of these calculations is based on three key elements: the assumption that the nucleus consists of A nucleons interacting via the strong interaction as characterized by the nucleon-nucleon potential, $V_{N N}$; the inference of this potential empirically (typically parameterized in terms of a meson exchange description of the nuclear force) from a fit to measured nucleon-nucleon phase shifts; and the addition of corrections for exchange current and leading relativistic effects in $V_{N N}$ and the nucleus. These calculations can be refined by carrying out more complete treatments of exchange currents and relativity, and their accuracy can be estimated by considering the theoretical uncertainties from the neglect of three-body forces, and from phase-shift equivalent potentials. The talk by Pandharipande [13] at this conference discusses these issues and describes the state of the art.

A long-standing problem in few-body physics has been the inability of theories that described the 3-body systems ${ }^{3} \mathrm{He}$ and ${ }^{3} \mathrm{H}$ to reproduce the experimental data on the twobody system (the deuteron). The clearest signature of this problem was the disagreement in the location of the diffraction minimum] in the deuteron's monopole form factor [14]. Available deuteron elastic scattering data had significant discrepancies and didn't cover an adequate range of $Q^{2}$, so it was uncertain whether the disagreement was in the data or due to an inadequacy of the theory.

A pair of recent experiments at Jefferson Lab have dramatically extended the range of $Q^{2}$ covered in elastic $e-d$ scattering, and greatly improved our information on this fundamental nuclear system. The first of these [15] measured elastic scattering at both forward and backward angles, which are most sensitive to the $A\left(Q^{2}\right)$ and $B\left(Q^{2}\right)$ structure functions, respectively. $A\left(Q^{2}\right)$, shown in Fig. 8, was measured to a momentum transfer of $6(\mathrm{GeV} / \mathrm{c})^{2}$, corresponding to a spatial resolution of $\sim 1 / 4 \mathrm{fm}$. Note that the cross section for the highest $Q^{2}$ point is $\sim 3 \times 10^{-41} \mathrm{~cm}^{2}$, a value typical of neutrino scattering experiments. Three theoretical calculations are also shown; that of Van Orden et al. [16] is in remarkable agreement with the data.

Because the deuteron is not a spin-zero object, the electric and magnetic elastic form factors are incoherent sums over the contributing $\mathrm{C} 0, \mathrm{M} 1$, and $\mathrm{C} 2$ multipoles. Fig. 9 displays the multipole contributions calculated for the deuteron $A\left(Q^{2}\right)$ shown above. A third, independent measurement is needed to separate the multipole contributions and infer the ground state charge and current distribution unambiguously.

The traditional solution is to measure $t_{20}$, the tensor polarization of the recoiling deuteron. The recent experiment of Kox and Beise [18] has extended our knowledge of $t_{20}$ to a $Q^{2}$ of $1.8(\mathrm{GeV} / \mathrm{c})^{2}$; these new data are shown in Fig. 10. The zero-crossing of $t_{20}$ is highly sensitive to the location of the diffraction minimum in the monopole (C0) form factor. The analysis of these new data is not yet complete, and the error bars shown are double the anticipated final error bars. However, it is already clear that the new data will determine the location of the minimum precisely, and that it is at a lower $Q^{2}$ value than was inferred from earlier data (in agreement with theoretical expectations). Also shown in the figure are the predictions for $t_{20}$ from a variety of conventional nuclear physics theories; surprisingly, the deuteron ground-state wave function is well described within the context of classical nuclear physics down to a distance scale of order $0.5 \mathrm{fm}$. A more detailed comparison between theory and experiment will be possible when the new 


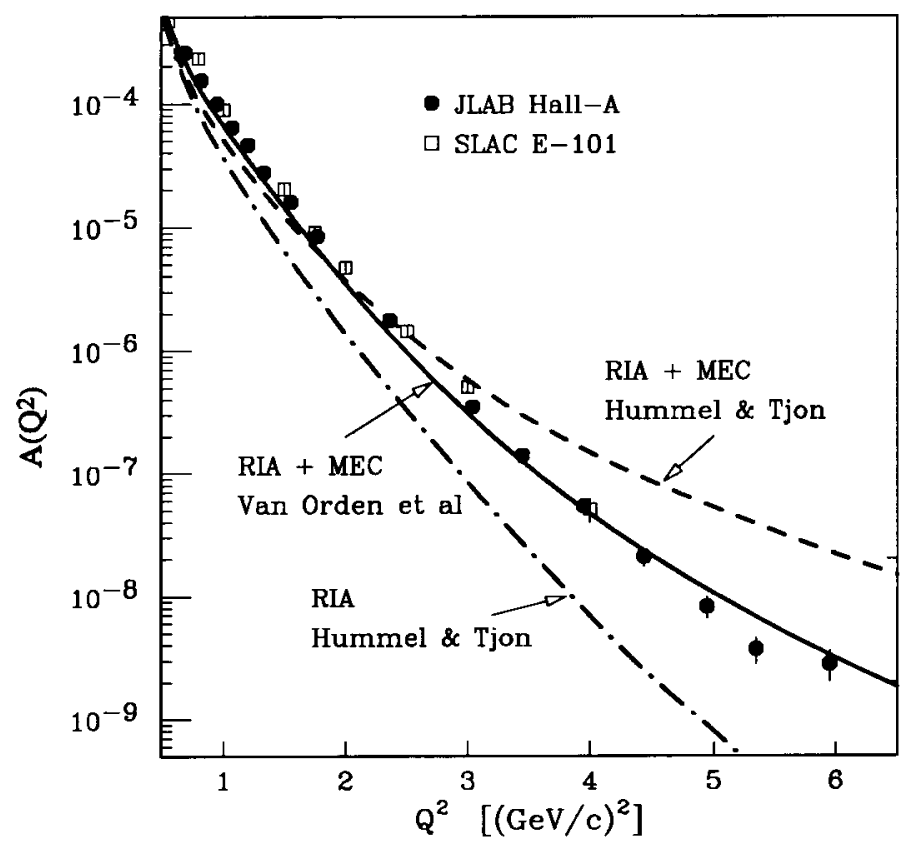

Figure 8. Preliminary Jefferson Lab data [15] on the deuteron $A\left(Q^{2}\right)$ and theoretical predictions based on nucleon degrees of freedom only.

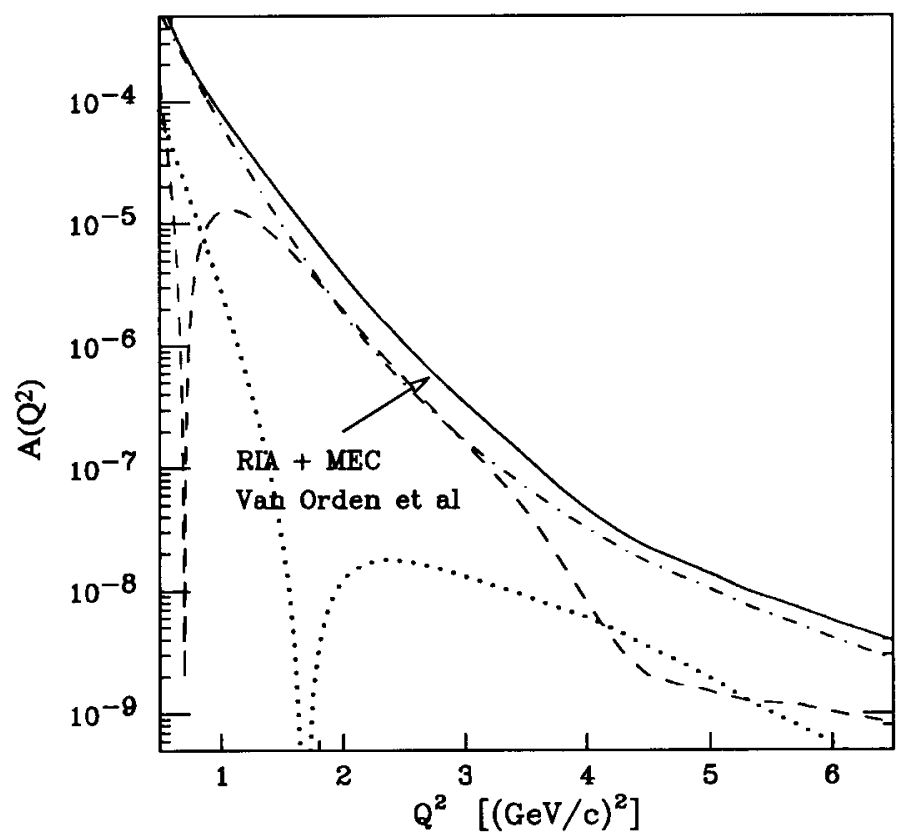

Figure 9. The multipole contributions to the theoretical $A\left(Q^{2}\right)$ of Van Orden, et al. [17], including C0 (dashed), M1 (dotted) and C2 (dash-dot). 
data have been finalized.

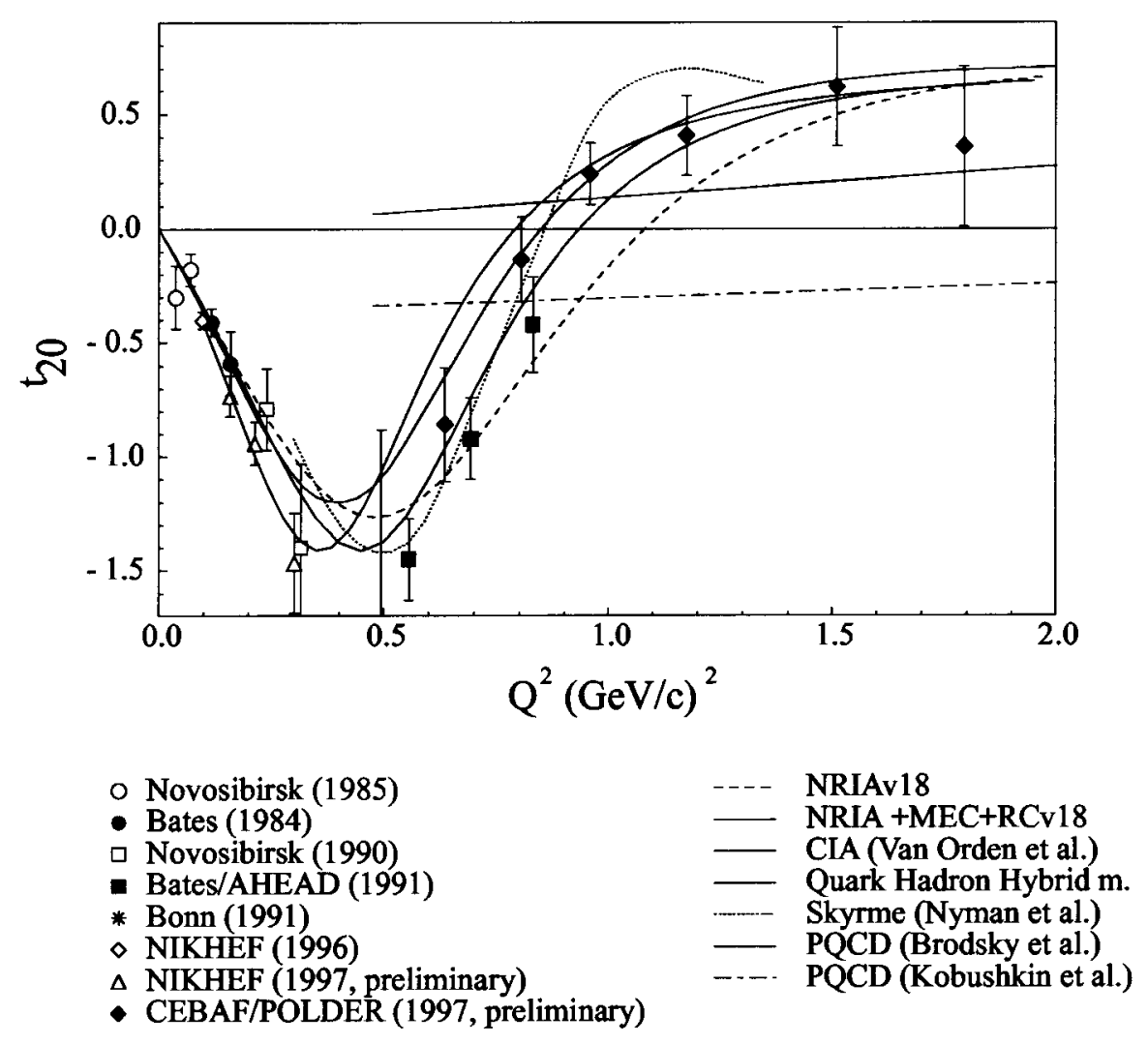

Figure 10. Tensor polarization in elastic e-d scattering

In contrast, the limitations of classical nuclear physics for describing the deuteron are evident from the recent measurement by Holt et al. [19] of the photodisintegration of the deuteron out to very high $(4 \mathrm{GeV})$ photon energies. The new $90^{\circ}$ data are shown in Fig. 11, where the cross sections have been multiplied by $s^{11}$ (where $s$ is the Mandelstam variable, equal to the square of the total energy in the center of mass frame) to account for the $\mathrm{pQCD}$ prediction of the $s^{-11}$ scaling behavior that should occur at very high energies.

The photon picks out very high momentum components of the wavefunction due to the momentum mismatch between the gamma and the final state. Also shown in this figure is the prediction of the classical nuclear physics theory of Lee [20]; it fails for $E_{\gamma} \geq 1 \mathrm{GeV}$. The experiment confirms the scaling-like behavior of the $90^{\circ}$ cross section first seen in an earlier SLAC experiment [21]. However, this behavior doesn't persist at more forward angles, implying that the true scaling regime has not been reached at $4 \mathrm{GeV}$. Two experiments to further explore this phenomenon are planned - one in which the experiment will be extended to $6 \mathrm{GeV}$, and a second in which the final state polarization will be measured. Since most theorists do not believe that the $s^{-11}$ behavior observed 


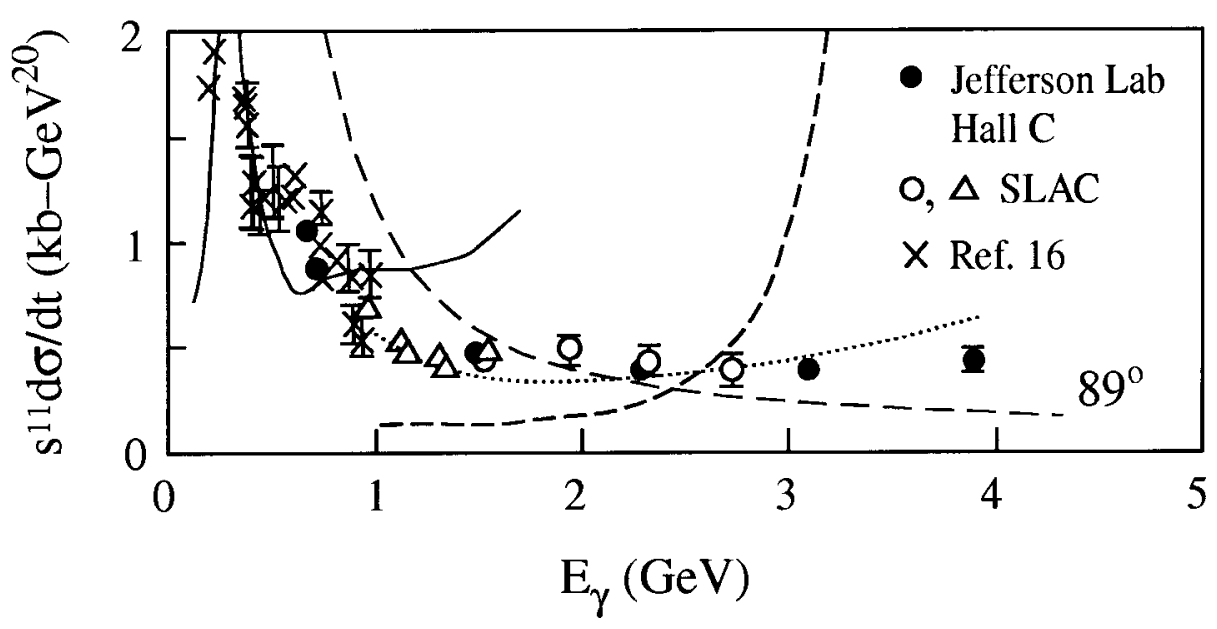

Figure 11. The photodisintegration of the deuteron at $90^{\circ}$ [19]. The prediction of Lee [20], based on nucleon degrees of freedom, is shown as the solid curve.

at these relatively low energies in the $90^{\circ}$ cross section is truly due to scaling at the six quark level, it is hoped that these experiments will shed further light on the underlying physics. It may be that we are seeing the onset of the transition from the nucleon degrees of freedom to the underlying quark degrees of freedom, with the photon interacting with a single quark.

Related experiments are underway aimed at understanding the origins of $V_{N N}$ and the exchange currents. At present we cannot distinguish a nucleon-nucleon interaction based on quark exchange from one based on meson exchange (see Fig. 12). However, we would expect these different mechanisms to have different predictions for interactions such as $\Lambda N, \Sigma N, \Delta N$, and $\Delta \Delta$. Experiments measuring these interactions are planned.

\subsection{Collective excitations in nuclei}

An example of the power of electron scattering coincidence measurements for the study of the highly collective giant resonance excitations is shown in Fig. 13, which displays data from a recent ${ }^{48} \mathrm{Ca}\left(e, e^{\prime} n\right)$ coincidence experiment [22] at Darmstadt. This experiment complements earlier $\left(e, e^{\prime} p\right)$ and $\left(e, e^{\prime} \alpha\right)$ giant resonance studies. The data show clear evidence of the coupling of the ${ }^{48} \mathrm{Ca}$ giant resonance to hole states in ${ }^{47} \mathrm{Ca}$, reflecting the structure of the ${ }^{48} \mathrm{Ca}$ giant resonance excitation. The shaded curve on the plot is the result of a statistical model calculation for the decay. The fact that this calculation underestimates the cross section for many of the observed final states is clear evidence that the decay has substantial direct and/or semi-direct components. This, in turn, tells us that the angular correlations for the decays contain a wealth of information about the structure of the giant resonance states; a detailed analysis is in progress. It would be very interesting to see this experimental technique extended to higher $q^{2}$, permitting the extraction of the transition change density for a giant multipole resonance for the first time. 


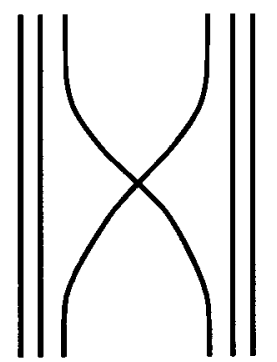

a)

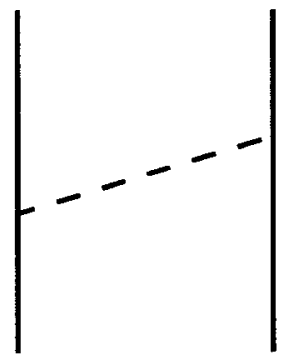

b)

Figure 12. Present experiments cannot distinguish between a nucleon-nucleon potential arising from a) quark exchange, and one arising from b) meson exchange.

\section{HOW IS THE NUCLEON CONSTRUCTED FROM QUARKS AND GLUONS?}

Now let me turn to the question of how the nucleon and other hadrons are constructed from quarks and gluons. QCD is well understood in the regime of very high energies (and very small distances), where it becomes asymptotically free. However, while we are convinced that QCD must form the basis for our understanding of nucleons and the nuclear force, that understanding is sketchy at best. The simple constituent quark model has done a rather remarkable job of describing basic characteristics of the nucleons and their observed excited states, but many open questions remain. For example, we are not sure of the effective degrees of freedom in the confinement regime as many of the states predicted by the constituent quark model have not been observed. We can't even explain why we find only $q \bar{q}$ and $q q q$ configurations in nature, rather than all possible color singlet combinations. In the naïve constituent quark model all the spin of the nucleon comes from the spin of the quarks, but experiments me asuring the spin structure functions tell us that the quarks carry only $\sim 1 / 3$ of the nucleon spin, with the remainder in the $q \bar{q}$ sea and the glue. More generally, the role of the $q \bar{q}$ sea in the nucleon is poorly understood. Another puzzle is that the quarks themselves carry only $\sim 1 / 2$ the nucleon mass; the rest must be in the glue, but we still have no direct evidence for the gluonic degree of freedom in hadron spectroscopy.

Many experiments investigating the QCD structure of the nucleons are in progress, including:

- tests of the QCD Hamiltonian in the confinement regime via comparisons of threshold $(\gamma, \pi)$ cross sections with the predictions of chiral perturbation theory $(\chi \mathrm{PT})$;

- measurements of meson and nucleon excited state structure and form factors, and of nucleon polarizabilities; 


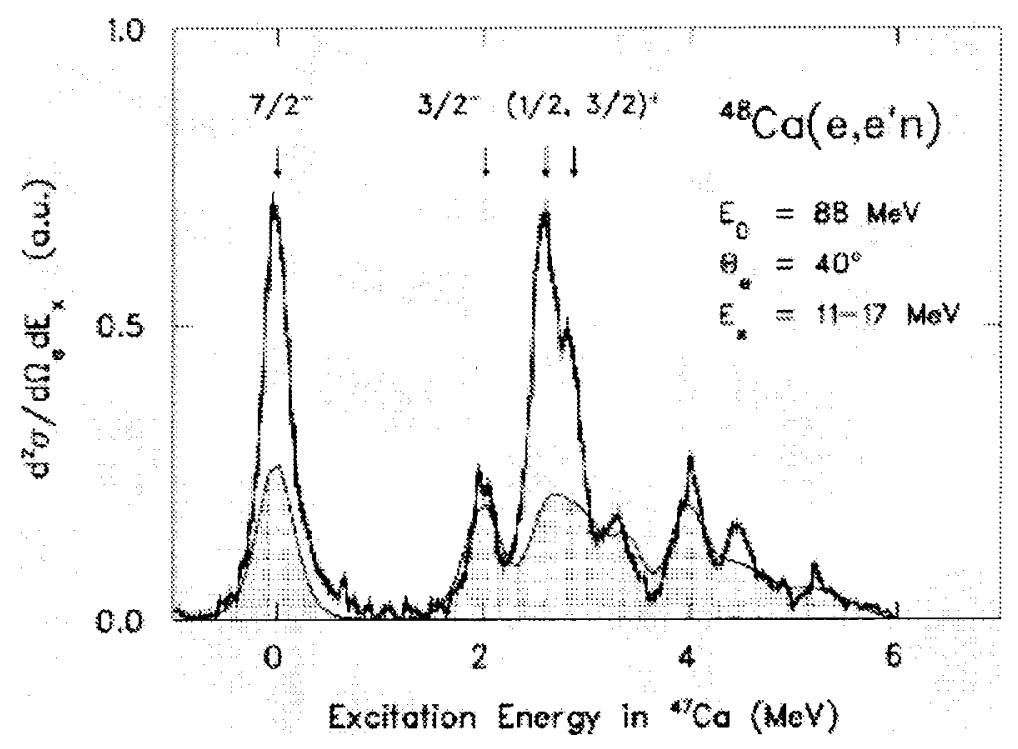

Figure 13. Excitation energy in the residual nucleus ${ }^{47} \mathrm{Ca}$ as measured using the ${ }^{48} \mathrm{Ca}\left(e, e^{\prime} n\right)$ reaction at Darmstadt [22] compared to the predictions of a statistical decay model (shaded area).

- studies of the strangeness distribution in the nucleon (sensitive to the character of the sea), of deep inelastic scattering structure functions (aimed at determining the momentum distributions of the quarks within the nucleon) [23], and tests of QCD-based sum rules and their $Q^{2}$ evolution;

- searches for $J^{P C}$ exotic mesons, which would provide direct evidence of gluonic excitation [24].

In the subsections that follow, I review some of the important issues in these areas, outline results from recent experiments, and describe the quality of data expected from new experiments in planning or underway.

\subsection{Nucleon form factors}

A key to understanding the structure of the neutrons and protons will be the precise measurement of the electro-weak form factors for their ground states, and the transition form factors for their excited states. The ground state form factors have many uses. They are fundamental ingredients in the classical model of nuclear physics - one must fold these form factors with the theoretical calculations of nuclear structure before comparing the theory with experiment. More importantly, these measurements determine the spatial structure of the charge density and of the current and magnetization densities of the nucleons, providing a stringent testing ground for theories constructing nucleons from quarks and gluons.

By measuring the weak neutral current form factors for the nucleons as well, we will be in a position to carry out a complete flavor decomposition of the nucleon form factors. 
(I.e., measurements of $G_{E}^{p}, G_{E}^{n}, G_{M}^{p}, G_{M}^{n}, G_{Z}^{p}$, and $G_{Z}^{n}$ can be combined to infer the flavorseparated form factors, $G_{E}^{u}, G_{E}^{d}, G_{E}^{s}$, and $G_{M}^{u}, G_{M}^{d}, G_{M}^{s}$, describing the distributions of charge and current associated with each quark flavor). These separated distribution functions will provide unique experimental insights into nucleon structure.

Additional insights will be gained from the measurement of the form factors of nucleons embedded in the nuclear medium. Changes in the form factors (estimated [25] to be as large as $10 \%$ ) have implications for phenomena as wide-ranging as nuclear binding, the equation of state for nuclear matter, and the EMC effect, and could provide important evidence of the precursor to the quark-gluon plasma.

\subsubsection{The proton electric form factor}

The recently completed experiment of Perdrisat et al. [26], which used the spin transfer technique to measure the ratio of the electric to the magnetic form factors of the proton to $Q^{2} \simeq 3.5(\mathrm{GeV} / \mathrm{c})^{2}$, provides a beautiful example of the quality of the data that is emerging. We now know unequivocally that the radial distribution of charge and magnetization in the proton differ significantly, and we have data on the proton electric form factor (using this experiment and the much better known magnetic form factor) that begins to distinguish among models for the proton's structure.

\subsubsection{The neutron electric form factor}

The neutron's electric form factor is particularly interesting. We know, of course, that the neutron has no net charge, but we also know (from measurements of neutron scattering from atomic electrons) that the neutron has a charge distribution, with positive charge in the interior and negative charge at large radii. It is not known whether this charge density arises from the neutron's pion cloud (see Fig. 14) or from an asymmetric distribution of the three valence quarks (ddu).

Experiments aimed at determining $G_{E}^{n}$ are difficult because it is not possible to construct a useable target of free neutrons; instead we must infer the neutron cross sections from measurements on the deuteron or ${ }^{3} \mathrm{He}$. We need detailed knowledge of the structure of these nuclei to extract $G_{E}^{n}$ reliably from the measured cross sections (the neutron is, in general, not at rest when struck, so the measured cross sections must be corrected for this motion). The difficulties are illustrated by the central plot in Fig. 14, which displays the available data on $G_{E}^{n}$. The Saclay data, which were obtained from elastic e-d scattering, depend strongly on the assumed nucleon-nucleon potential for their interpretation; this dependence manifests itself as a nearly constant scaling factor for the extracted $G_{E}^{n}$ values. Data from the first generation of exclusive reactions aimed at measuring $G_{E}^{n}$ shows systematic differences between the values extracted from experiments using ${ }^{3} \mathrm{He}$ and those using deuteron targets. Complementary experiments at a number of laboratories are addressing the relevant structure questions. With these questions answered, we can anticipate the combination of existing data, consistently reanalyzed, and planned new experiments (bottom plot in Fig. 14), which will use the spin transfer technique to extend the $G_{E}^{n}$ data to $Q^{2} \sim 2(G e V / c)^{2}$ with very small error bars, will provide a difinitive measurement of $G_{E}^{n}$. These results, together with the recent accurate determination [28] of the neutron's magnetic form factor, $G_{M}^{n}$, will provide stringent tests for models of the neutron. 

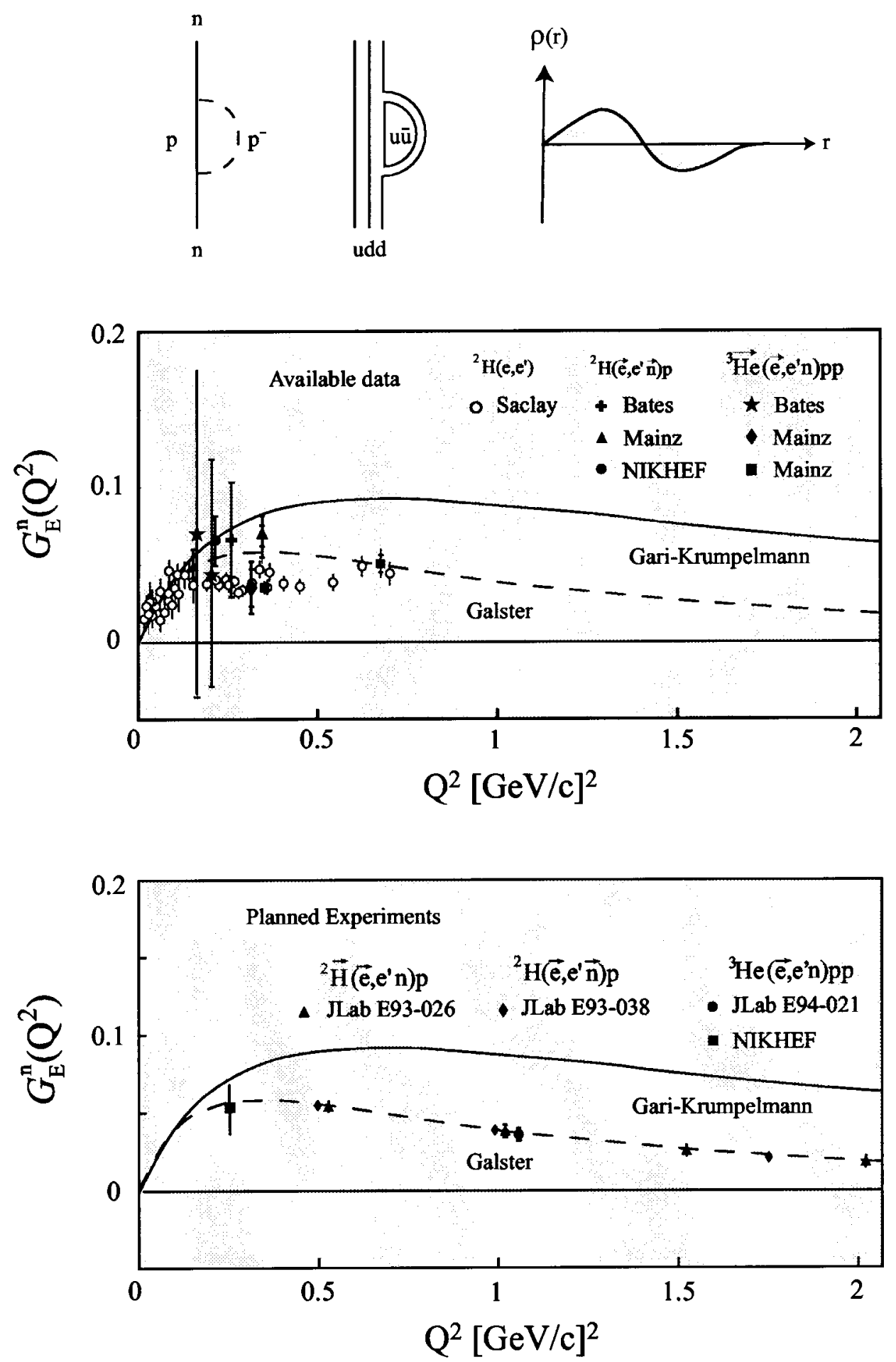

Figure 14. Top: Diagrams giving rise to $\left(G_{E}^{n}\right)$ in the conventional and quark pictures of the neutron (left) and a sketch (right) of $\rho_{n}(r)$ consistent with our limited knowledge of $G_{E}^{n}$. Center: Theoretical estimates of $G_{E}^{n}$, together with data from previous experiments. Bottom: Expected data from planned experiments [27]. 


\subsubsection{The strangeness distribution of the proton}

Just as the fact that the neutron has no net electric charge doesn't imply a zero charge distribution within the neutron, the fact that the proton has no net strangeness doesn't imply a zero strangeness distribution. Measurement of that distribution, which arises from the presence of virtual $s \bar{s}$ pairs, should provide us with important new information on the structure of the proton, and, in particular, on the $q \bar{q}$ sea. This is shown schematically in Fig. 15. The analogy between the $s \bar{s}$ cloud of the proton and the pion cloud of the neutron are evident from a comparison of these diagrams with those of Fig. 14. However, the strangeness distribution is uniquely sensitive to the nucleon sea because, unlike the case of the $u \bar{u}$ pairs in the neutron, the $s \bar{s}$ pairs in the proton can only arise from virtual excitation of the glue.

The basic idea behind the experimental approach to measuring these new quantities is to assume that electroweak theory is correct, and that the electro-weak mixing angle $\left(\sin ^{2} \theta_{W}\right)$ has been determined from other experiments. Since the weak neutral current contribution to the total electroweak interaction is small $\left(10^{-5}\right.$ to $10^{-6}$ is typical $)$, it can only be measured via parity-violation experiments. The parity-violating asymmetry is proportional to the ratio of the weak form factor to the total (weak plus electromagnetic) form factor; since the electromagnetic form factors are known, the weak neutral current form factor can be inferred from the measured asymmetry.

Just as the measurement of the magnetic form factor of the proton at very low $Q^{2}$ is primarily sensitive to the proton's magnetic moment $\mu_{p}$, a low $Q^{2}$ measurement of the weak magnetic form factor is primarily sensitive to the proton's weak magnetic moment, $\mu_{Z}$. A measurement of $\mu_{Z}$, taken together with the known electromagnetic moments, will permit the decomposition of the proton's electro-weak magnetism into the contributions from the different quark flavors: $\mu_{p}^{u}, \mu_{p}^{d}$, and $\mu_{p}^{s}$. The first phase of the SAMPLE experiment at MIT/Bates [29], which was carried out at a momentum transfer of $0.1(\mathrm{GeV} / \mathrm{c})^{2}$, has measured $\mu_{Z}=0.34 \pm 0.11 \mu_{N}$. A second run, planned for next summer, is expected to halve the error bar.

An experiment aimed at investigating the spatial distribution of the weak neutral current structure of the proton in greater detail is the HAPPEX experiment [30] at Jefferson Lab. This experiment is at a $Q^{2}$ of $0.48(\mathrm{GeV} / \mathrm{c})^{2}$, and involves a measurement at forward angles that is sensitive to a combination of the "electric" and "magnetic" weak form factors: $G_{E}^{s}+0.39 G_{M}^{s}$. The result is shown in Fig. 15, together with the predictions of a number of theoretical predictions. The measured asymmetry of $-14.5 \pm 2.2 \mathrm{ppm}$ is consistent with the asymmetry predicted by the electroweak standard model with no additional contribution from strange quarks. These data are consistent with the strangeness distribution - averaged over a distance scale of order $1 \mathrm{fm}$ - being zero, and exclude some models convincingly. One simple interpretation of the data is that the $s \bar{s}$ pairs stay relatively close to each other in the nucleon. A second run of this experiment, planned for 1999, will halve the error bar.

\subsubsection{The excited states of the nucleons}

One key problem in our understanding of the nucleon is that of the missing states in its excitation spectrum. Fig. 16 displays both experimentally identified excited states of the proton and the predictions of the quark model [31]. Most of the observed states 

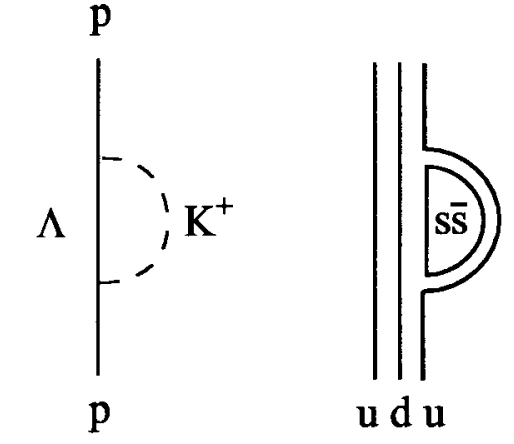
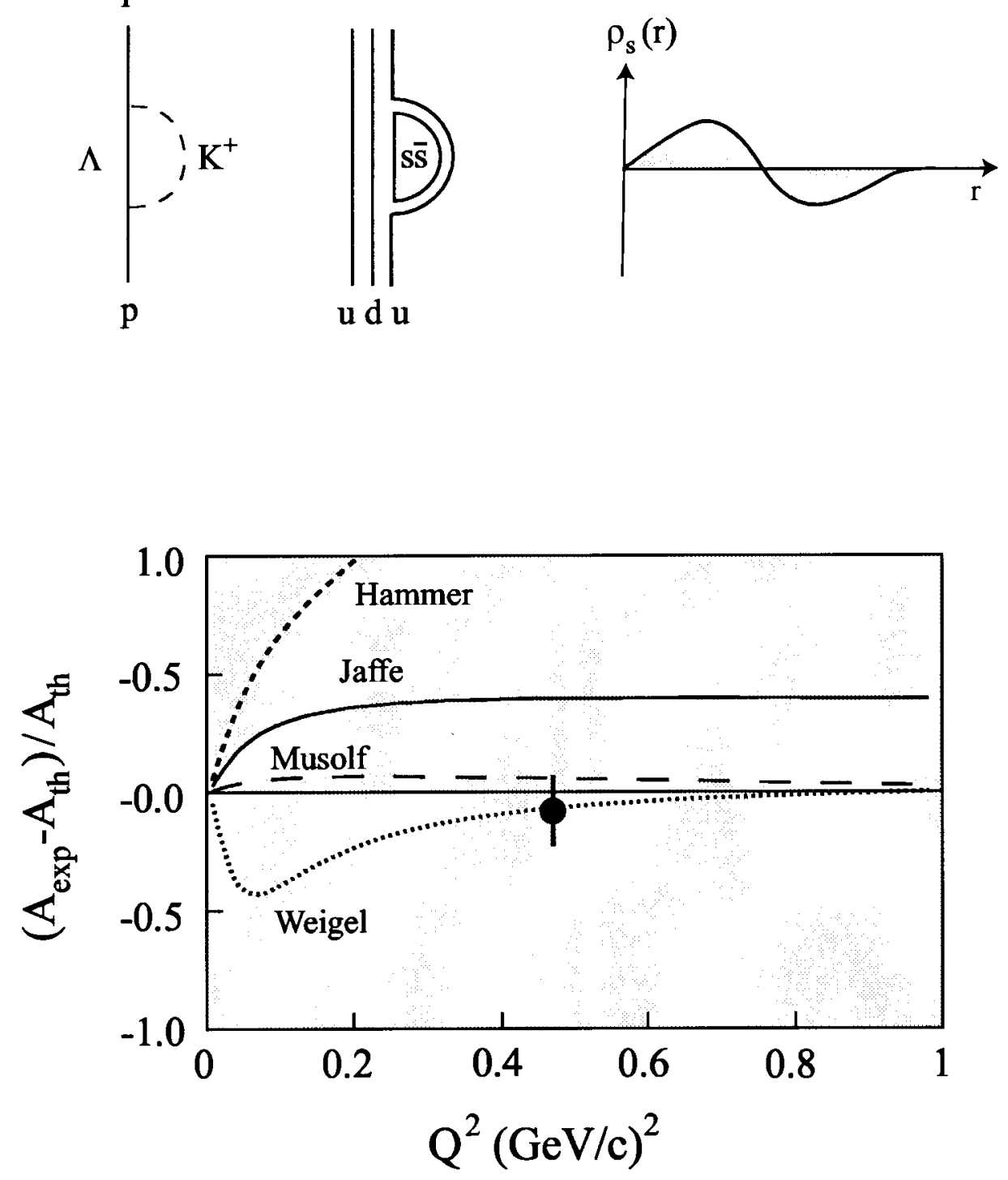

Figure 15. Top Figure: Diagrams giving rise to the strangeness form factor in the conventional and quark pictures of the neutron (left) and a sketch of a possible strangeness distribution (right). Bottom Figure: the HAPPEX result for the scattering asymmetry for polarized electrons from protons. Zero corresponds to the asymmetry predicted from the known nucleon form factors in the absence of strange quark effects [30]. 
were found using the $\pi \mathrm{N}$ reaction. About half of the predicted states have not been found experimentally. It has been noted that the missing states would not be present in a diquark-quark version of the quark model. Since the missing states are predicted to couple only weakly to the $\pi \mathrm{N}$ channel, a search for them in the photon channel is one of the main foci of the $\mathrm{N}^{*}$ program of the CLAS detector in Hall B at Jefferson Lab.

As has been the case, e.g., for atoms, we anticipate that the detailed spectrum will identify the appropriate low energy effective degrees of freedom in the proton.

The experimental difficulties faced by the $\mathrm{N}^{*}$ program are evident from Fig. 17, which shows an inclusive electron scattering spectrum for the proton. While three excitations are obvious to the naked eye, a detailed phase shift analysis of data is needed to infer the energies and quantum numbers of the remaining states. The difficulties arise from the fact that the states are separated in energy by much less than their widths.

Typical data obtainable with CLAS in a few days of running is shown in Fig. 18. The data are plotted as a function of $W$, the invariant mass deposited in the system by the inelastically scattered electron, and $M_{x}$, the observed missing mass in the reaction. Note that substantial strength is observed in the "missing resonance" region, implying that there is significant coupling of states in this region to the photon channel, as expected theoretically.

The quantity of data that can be obtained using this new instrument is impressive; in its first few months of operation it roughly doubled the world's supply of data on electroand photo-production from the proton. The quality of the data that can be obtained in individual reaction channels is shown in Fig. 19, compared with data previously available. The new data has an accuracy that will be of great value for the required phase shift analysis.

Just as has been the case for the study of nuclear structure with electrons, the full power of electron scattering will be available only when one measures the transition form factors for the excited states of the nucleon, providing the kind of detailed information on the spatial structure of the states that will distinguish cleanly between theories of nucleon structure. The relatively primitive state of our ability to explain such data can be seen from results to date on the transition form factor for the nucleon's first excited state, the delta. The excitation is dominantly M1, arising from a quark spin flip, but small $\mathrm{C} 2$ and E2 contributions are sensitive to the detailed properties of the proton, such as its deformation. The experimental situation is complicated by the fact that the delta is not an isolated excitation (see Fig. 17), but lies on a background coming from both the continuum and the excitation of higher-lying states.

Fig. 20 displays new data on photo-excitation from LEGS [35] and Mainz [36], and on electro-excitation from MIT/Bates [37] and Jefferson Lab [38], together with data from previous measurements. The scatter of the data in this figure is not due inconsistencies among the measured cross sections - rather it arises from the fact that the approaches used to extract the transition form factor from the experimental data have been inadequate (typically using only s and p-waves in the analysis and either ignoring or assuming small contributions from interfering processes that are not well-measured). There is clear evidence for the inadequacies of these assumptions from the MIT/Bates experiment. Understanding the details of the processes associated with excitations in the region of the first excited state of the proton, and a detailed, consistent analysis of the delta can be 


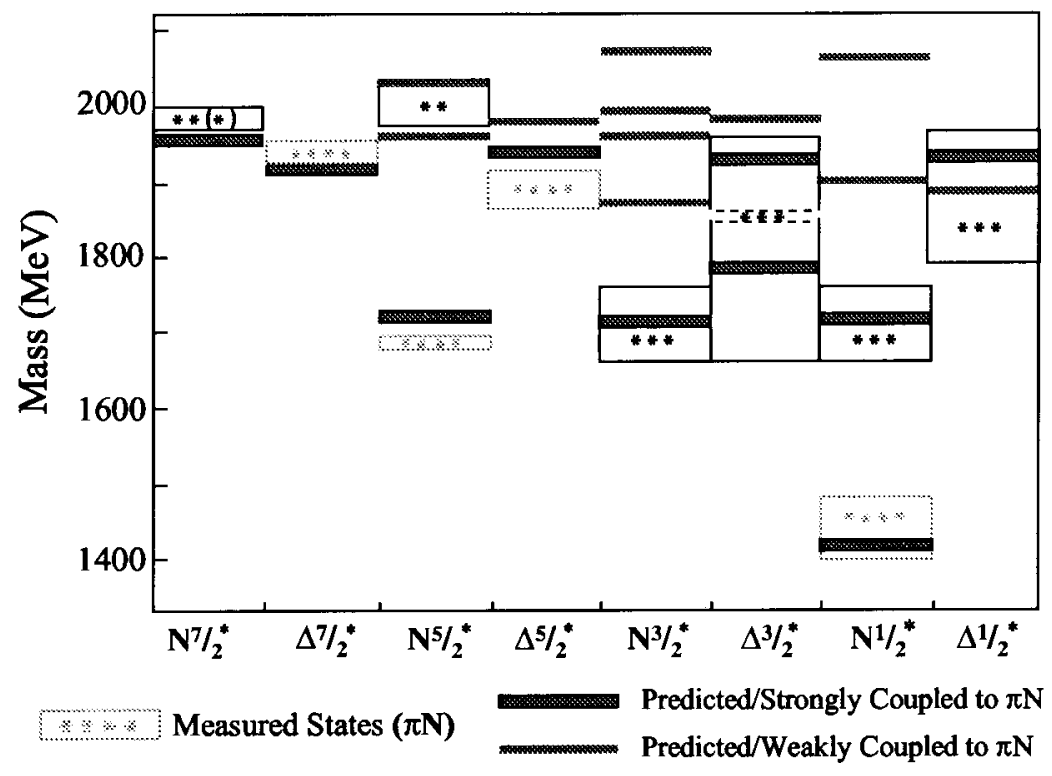

Figure 16. The predictions of the constituent quark model for the excited states of the proton. Experimentally identified states are shown as light boxes, with the number of asterisks in the box corresponding to the reliability of the observation as determined by the Particle Data Group.

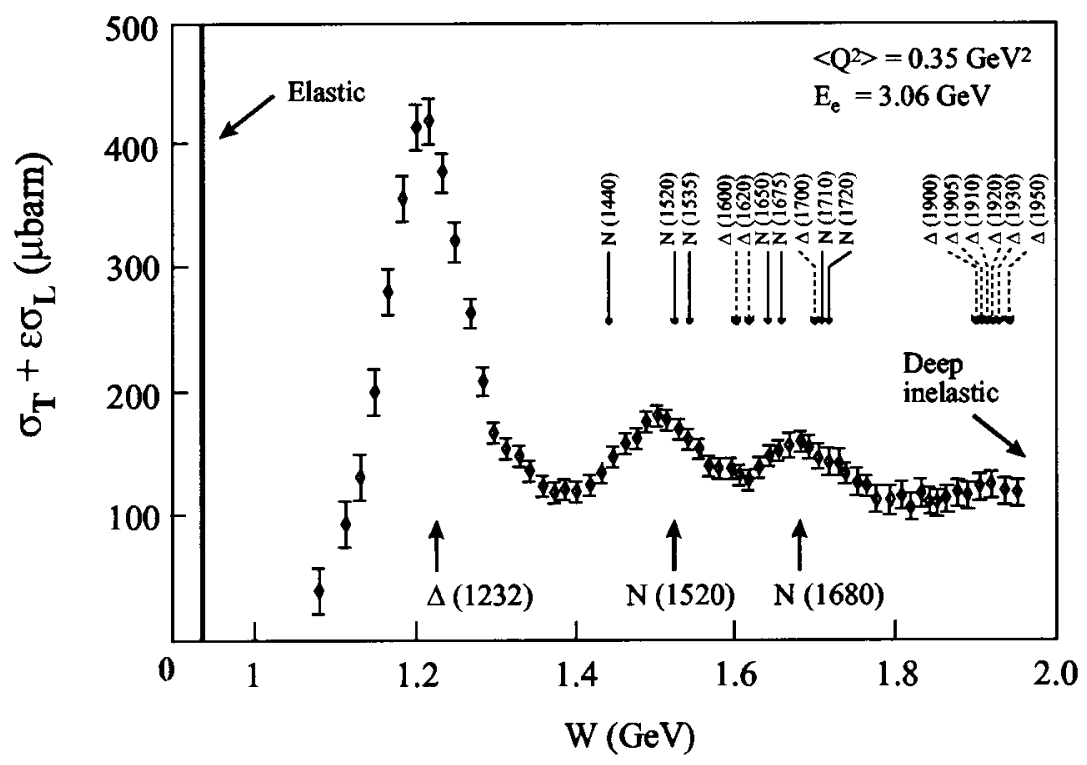

Figure 17. A typical spectrum for inclusive electron scattering from the proton [32]. The three easily identifiable resonances are marked from below, while the energies of the additional excited states predicted by the symmetric constituent quark model are marked from above. The observed states are identified with a solid arrow, while the unobserved states are identified with a dashed arrow. 


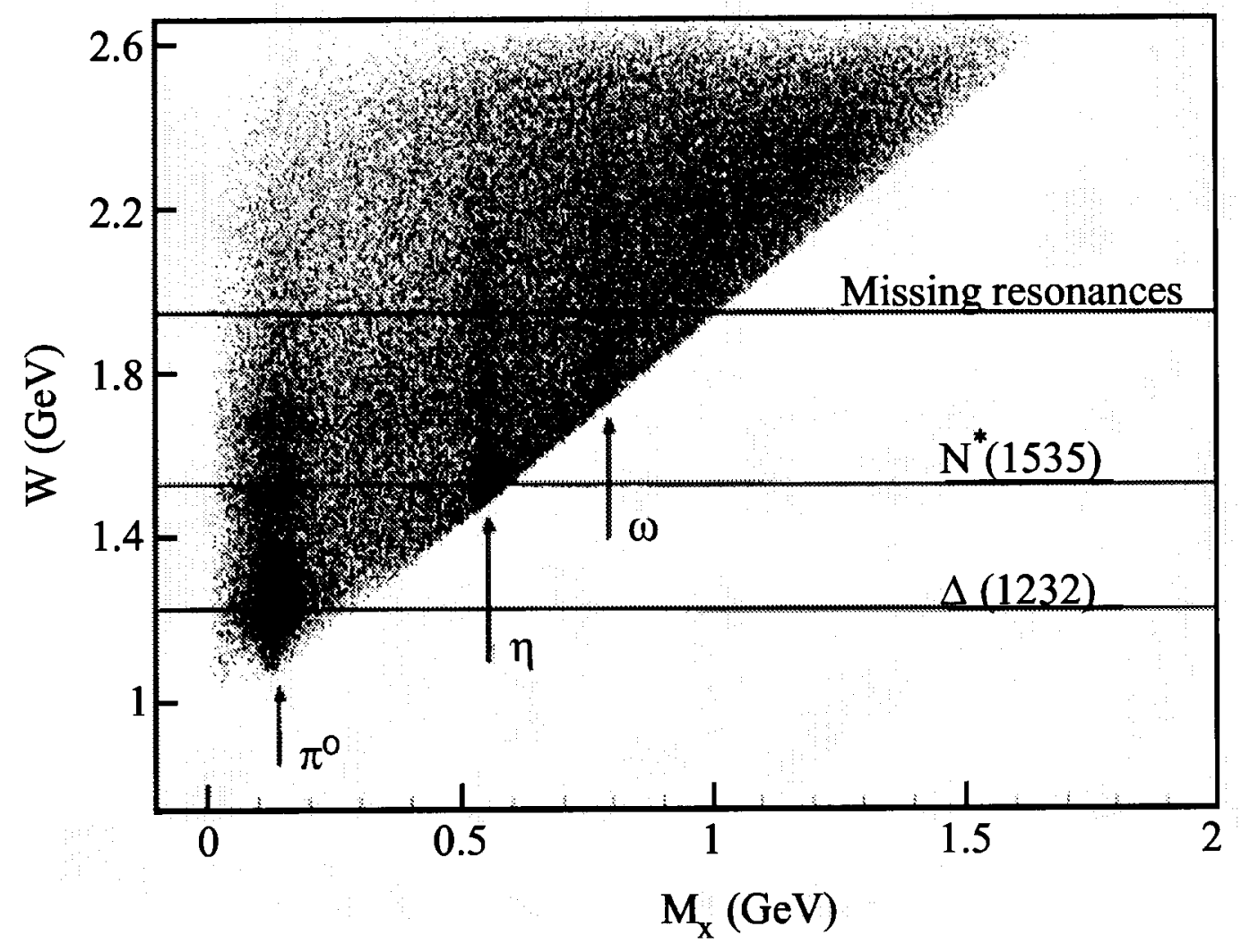

Figure 18. Data [33] for the reaction $e p \rightarrow e^{\prime} p x$ at $E_{e}=2.4 \mathrm{GeV}$. The data are plotted as a function of $W$, the invariant mass deposited in the system by the inelastically scattered electron, and $M_{x}$, the observed missing mass in the reaction. 

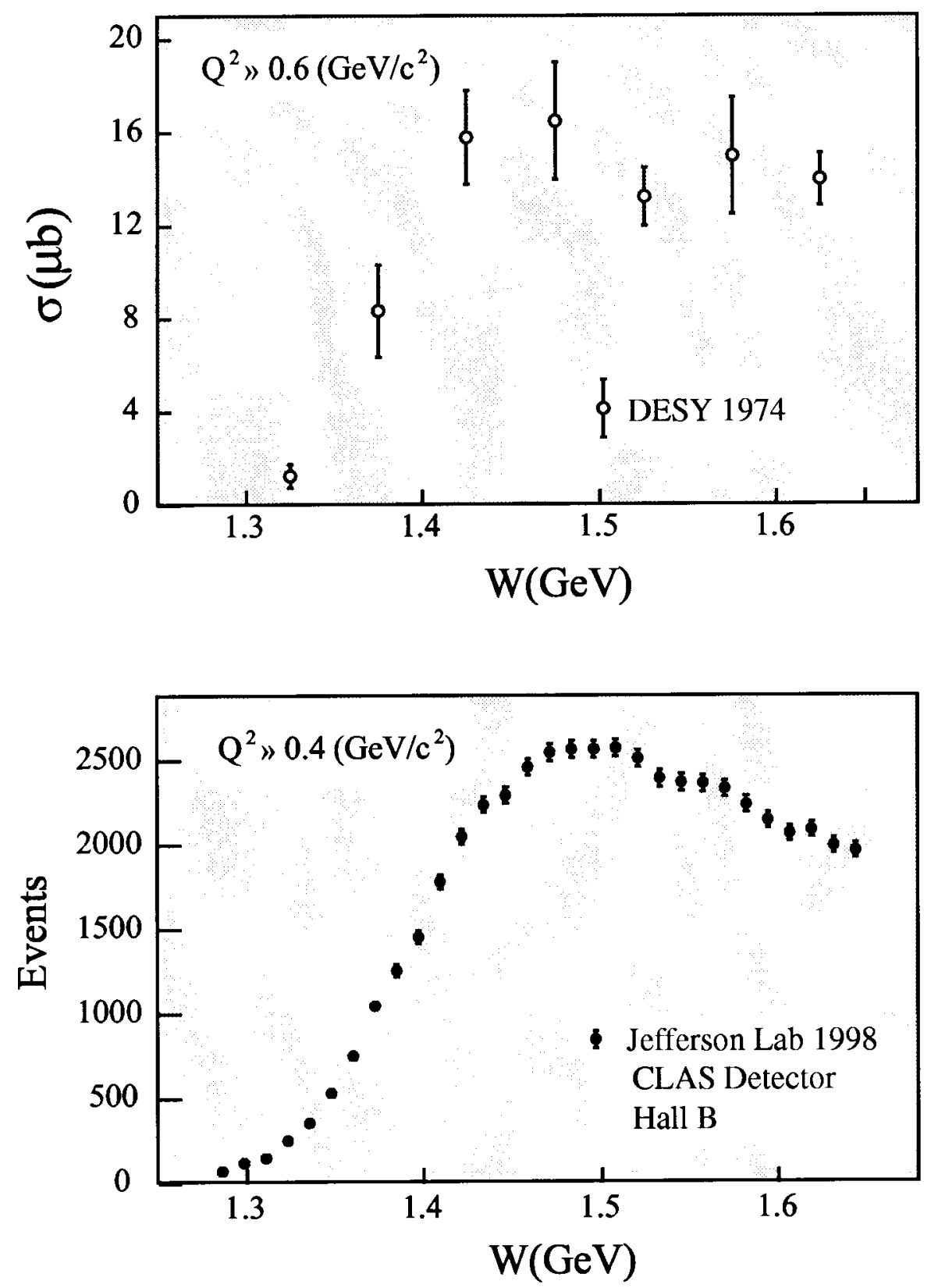

Figure 19. Data for the reaction $e p \rightarrow e^{\prime} \Delta^{++} \pi^{-}$obtained [33] at $E_{e}=2.4 \mathrm{GeV}$ (bottom) are compared with previous results (top) [34]. 


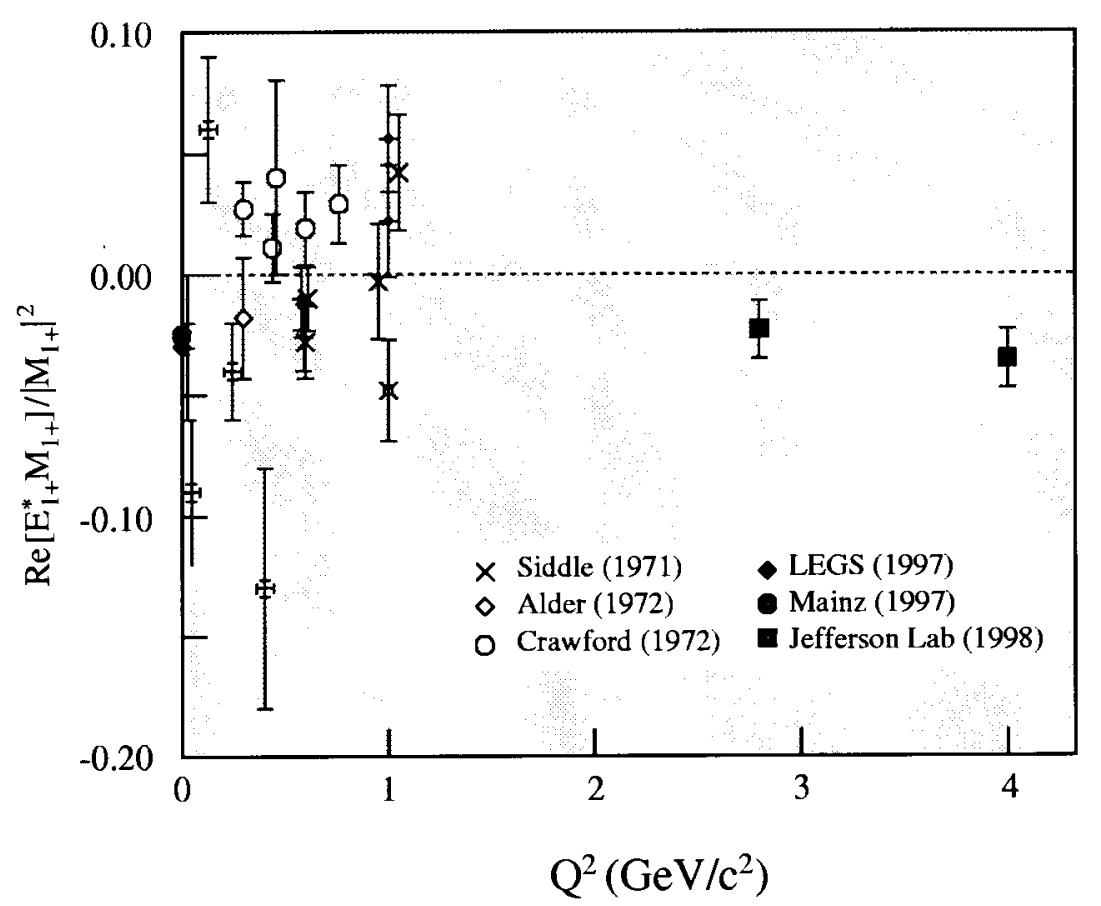

Figure 20. Data on the form factor for the excitation of the $\Delta$. [39]

expected to resolve the ambiguities in the present data and provide new insights into the structure of the proton.

\section{CONCLUSIONS}

The new generation of electron accelerators and experimental technology are making dramatic progress in accessing the details of nuclear dynamics, testing the limits of classical nuclear theory, and exploring the QCD basis of the strong interaction and the structure of nucleons and nuclei. We can expect the upcoming years to be extraordinarily fertile as we bring these new tools to bear on addressing these important problems.

\section{ACKNOWLEDGEMENTS}

It is a pleasure to acknowledge the contributions of my colleagues at laboratories around the world for providing results prior to publication and for many fruitful discussions. It is also a pleasure to acknowledge a careful reading of this manuscript and many thoughtful suggestions for its improvement by Kees de Jager, Bernard Frois, and Nathan Isgur.

\section{REFERENCES}

1. E. M. Lyman, A. O. Hanson, and M. B. Scott, Phys. Rev. 84 (1951) 626.

2. R. Hofstadter and R. W. McAllister, Phys. Rev. 98 (1955) 217.

3. B. Frois, C. N. Papanicolas and S. E. Williamson, Modern Topics in Electron Scattering, B. Frois and I. Sick, eds. (World Scientific, Singapore, 1991). 
4. E. N. M. Quint, Ph. D. thesis, Univ. of Amsterdam (1988); E. N. M. Quint et al., Phys. Rev. Lett. 57 (1986) 186; and Phys. Rev. Lett. 58 (1987) 1088.

5. V. R. Pandharipande, C. N. Papanicolas, and J. Wambach, Phys. Rev. 53 (1984) 473.

6. C. Marchand et al., Phys. Rev. Lett. 60 (1988) 1703.

7. L. Lapikas, Nucl. Phys. A553 (1993) 297c.

8. K. I. Blomqvist et al. Phys. Lett. B421 (1998) 71.

9. T. Ohmura et al., Prog. Theor. Phys. 15 (1956) 222.

10. S. Pieper et al., Phys. Rev. C 46 (1992) 1741.

11. D. L. Groep et al., proceedings of this conference.

12. J. Golak et al., Phys. Rev. C 51 (1995) 1638.

13. V. R. Pandharipande, proceedings of this conference.

14. H. Henning, J. Adam, P.U. Sauer, and A. Stadler, Phys. Rev. C52 (1995) 471.

15. G. G. Petratos, proceedings of this conference; and L. C. Alexa, et al., Phys. Rev. Lett. 82 (1999) 1374.

16. J. W. van Orden, B. N. Devine, and F. Gross, Phys. Rev. Lett. 75 (1995) 4369.

17. G. G. Petratos, private communication

18. M. Garçon, proceedings of this conference.

19. C. Bochna et al., Phys. Rev. Lett. 81 (1998) 4576.

20. T.-S. H. Lee, Argonne National Laboratory Report No. PHY-5253-TH-88; and T.S. H. Lee, in Proc. Int. conf. on Medium and High Energy Nuclear Physics, Taipei, Taiwan, 1988, (World Scientific, Singapore, 1988) p. 563.

21. J. E. Belz et al., Phys. Rev. Lett. 74 (1995) 646.

22. S. Strauch, Ph. D thesis, Tech. Univ. Darmstadt (1998); and P. von Neumann-Cosel and A. Richter, private communication.

23. A. Magnon, proceedings of this conference.

24. A. Zenoni, proceedings of this conference.

25. See, e.g., Electromagnetic Form-Factors of the Bound Nucleon, D.H. Lu et al., University of Adelaide preprint ADP-98-39-T311, July 1998.

26. G. Quéméner, procedings of this conference.

27. C. W. de Jager, private communication.

28. H. Anklin et al., Phys.Lett. B428 (1998) 248.

29. B. Mueller et al., Phys. Rev. Lett. 78 (1997) 3824.

30. A. Aniol et al. Phys. Rev. Lett. 82 (1999) 1096.

31. N. Isgur and G. Karl, Phys. Rev. D23 (1981) 817.

32. V. Burkert, private communication.

33. CLAS e1 collaboration, w/ thanks to L. Elouadrhiri, private communication.

34. P. Joos et al., Phys. Lett. 52B (1974) 481, and Phys. Lett. 62B (1976) 230.

35. G. Blanpied et al., Phys. Rev. Lett 79 (1997) 4337, and A. Sandorfi (private communication)

36. R. Beck et al., Phys. Rev. Lett. 78 (1997) 606.

37. C. Mertz et al., proceedings of this conference; and C. N. Papanicolas, private communication.

38. V. V. Frolov et al., Phys. Rev. Lett. 82 (1999) 45.

39. C. Vellides and C. N. Papanicolas, private communication. 